\title{
Análise das taxas de infecção e duração de cateteres de hemodiálise de curta e longa permanência em hospital de ensino
}

\author{
Analysis of infection rates and duration of short and long-term hemodialysis catheters \\ in a teaching hospital
}

\author{
Seleno Glauber de Jesus-Silva ${ }^{1}$ (D), Jennifer dos Santos Oliveira², Karine Tobias França Ramos², \\ Luciene Azevedo Morais ${ }^{3}$, Melissa Andreia de Moraes Silva ${ }^{1,2}$ (D), Arturo Eduardo Krupa ${ }^{1}$, Rodolfo Souza Cardoso
}

\begin{abstract}
Resumo
Contexto: Os cateteres venosos centrais para hemodiálise (CVCH) de curta permanência (CCP) e cateteres tunelizáveis de longa permanência (CTLP) são fundamentais para a terapia hemodialítica. Entretanto, há escassa casuística nacional acerca da incidência de complicações desses dois tipos de cateteres. Objetivos: Analisar as complicações e tempo de duração de CVCHs em centro de hemodiálise de hospital de ensino. Métodos: Foi feito um estudo unicêntrico, longitudinal e retrospectivo de 115 pacientes consecutivos submetidos a implante de cateteres para hemodiálise (67 CCP e 48 CTLP) em um período de 2 anos, com análise de sobrevida geral, perviedade, perda do acesso e incidência de complicações. Resultados: Sessenta por cento eram do sexo masculino e a média de idade foi de 62 anos. O principal sítio de punção foi a veia jugular interna direita. Hipertensão arterial sistêmica estava presente em $95 \%$ dos casos. A mediana de permanência do cateter foi de 50 dias (CCP) versus 112 dias (CTLP; $p<0,0001$ ). Não houve diferença na sobrevida global. Infecção relacionada ao cateter apresentou maior incidência nos CCP, sendo Staphylococcus sp. o microrganismo mais encontrado. A taxa de infecção por 1.000 dias foi maior nos CCP em relação aos CTLP (16,7 eventos/1.000 dias versus 7,0 eventos/1.000 dias, respectivamente). Baixa renda foi o único fator relacionado a maior incidência de infecção. Conclusões: $O$ tempo de permanência dos CTLP foi significativamente maior que os CCP, porém ainda assim abaixo dos valores relatados na literatura e sem impacto na sobrevida global. Baixa renda foi um fator associado a infecção de cateter.
\end{abstract}

Palavras-chave: diálise renal; infecções relacionadas a cateter; cateteres de demora.

\begin{abstract}
Background: Short-term (ST) and long-term tunneled (LTT) central venous catheters for hemodialysis (CVCH) are critical for hemodialysis therapy. However, few studies have been conducted in Brazil to investigate the incidence of complications with these two types of catheters. Objectives: To analyze complications and duration of $\mathrm{CVCH}$ in a hemodialysis center at a teaching hospital. Methods: Single-center, longitudinal, and retrospective study of 115 consecutive patients undergoing hemodialysis catheter placement (67 ST and 48 LTT) over a 2-year period, analyzing overall survival, patency, loss of access, and incidence of complications. Results: Sixty percent of the patients were male and mean age was 62 years. The most common puncture site was the right internal jugular vein. Systemic arterial hypertension was present in $95 \%$ of cases. Median catheter in-place duration was 50 days (ST) vs. 112 days (LTT; $p<0.0001$ ). There was no difference in overall survival. Incidence of catheter-related infection was higher in ST $\mathrm{CVCH}$, with Staphylococcus sp. the microorganism most often found. The infection rate per 1000 days was higher in ST than in LTT catheters (16.7 events/1000 days vs. 7.0 events/1000 days). Low income was the only factor related to higher incidence of infection. Conclusions: The in-place duration of long-term catheters was significantly longer compared to short-term CVCH, but still below the values reported in the literature and without impact on overall survival. Low income was a factor associated with catheter infection.
\end{abstract}

Keywords: renal dialysis; catheter-related infections; indwelling catheters.

Como citar: Jesus-Silva SG, Oliveira JS, Ramos KTF, et al. Análise das taxas de infecção e duração de cateteres de hemodiálise de curta e longa permanência em hospital de ensino. J Vasc Bras. 2020;19:e20190142. https://doi. org/10.1590/1677-5449.190142

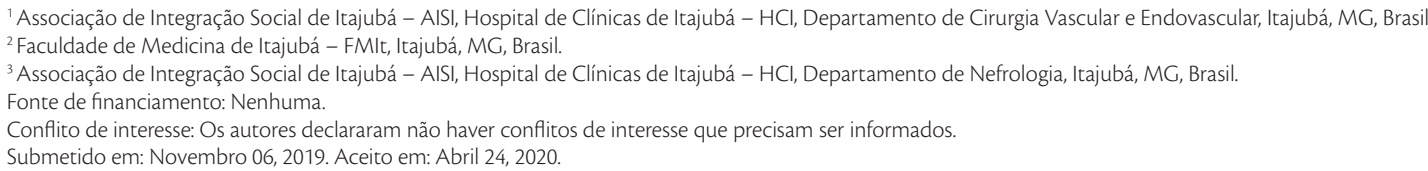




\section{INTRODUÇÃO}

A hemodiálise é um recurso amplamente utilizado no tratamento da insuficiência renal crônica terminal e envolve vários aspectos críticos para a sua execução, como o acesso vascular. Apesar de ser uma modalidade que, em geral, mantém a qualidade de vida do paciente, ela carrega consigo altos índices de complicação e internações, com taxa de mortalidade chegando a 10 a $25 \%$ por ano ${ }^{1}$. Embora o acesso ideal para a manutenção de hemodiálise a longo prazo seja a fístula arteriovenosa (FAV) ou a fístula com prótese, a realidade no Brasil é o uso, por grande parte dos pacientes, de cateteres venosos centrais para hemodiálise $(\mathrm{CVCH})$, com uma prevalência de até $20,5 \%{ }^{2}$. O uso de CVCHs tem aumentado, ao mesmo tempo em que a população envelhece e mais pacientes com poucas opções de confecção de FAV iniciam a hemodiálise.

Os cateteres não tunelizáveis de curta permanência (CCP) representam uma opção confiável nas situações que requerem início imediato à terapia de substituição renal e que não possuam acesso definitivo, mas que devem ser removidos o mais rápido possível, devido ao alto risco de complicações infecciosas ${ }^{3}$. Nos casos de impossibilidade de confecção de uma FAV, seja por ausência de veia adequada ou por debilidade clínica, os cateteres tunelizáveis de longa permanência (CTLP) são opções consideradas mais duradouras e com menores complicações ${ }^{4}$. O uso de CCP por mais de 3 meses é indicador negativo de qualidade dos serviços de hemodiálise no Brasil ${ }^{5}$.

Diversas complicações estão relacionadas aos CVCHs, incluindo as de implantação (hematomas, pseudoaneurismas, pneumotóraces), tromboses do cateter e de veias centrais, além da mais grave de todas, a infecção relacionada ao cateter. Esta última é associada a elevadas taxas de internação, custo e mortalidade ${ }^{6}$. Diversos fatores foram associados à perda dos $\mathrm{CVCHs}$, como idade avançada, história de múltiplos acessos prévios, grau de escolaridade, qualidade da manipulação do dispositivo pela equipe de enfermagem, diabetes, imunodepressão, entre outros $^{7,8}$.

Apesar do grande conhecimento disponível na literatura estrangeira acerca da evolução dos $\mathrm{CVCHs}$, poucos estudos regionais abordaram comparativamente suas taxas de permanência e de complicações. São necessárias, portanto, casuísticas que definam se os resultados de vida real em centro de hemodiálise brasileiros são comparáveis aos previstos na literatura, de forma a diagnosticar e corrigir possíveis falhas. $\mathrm{O}$ presente estudo teve por objetivo analisar, em centro de hemodiálise de hospital de ensino, as diferentes evoluções dos CCP e CTLP no que diz respeito a durabilidade, complicações infecciosas e não-infecciosas e fatores de risco associados.

\section{MÉTODOS}

Foi feito um estudo retrospectivo, observacional e longitudinal, no qual foram analisados, por meio de revisão de prontuários, 115 pacientes consecutivos submetidos a implante de CVCH (67 CCP e 48 CTLP) em serviço de terapia renal substitutiva de hospital de ensino quaternário, no período de janeiro de 2016 a janeiro de 2018. O estudo foi aprovado pelo Comitê de Ética em Pesquisa da instituição sob parecer $n^{\circ}$ 2.170.323.

O cálculo da amostra foi baseado na diferença estimada de permanência do $\mathrm{CVCH}$ em 30 dias (60\% para CCP e $90 \%$ para CTLP), poder de teste de $80 \%$ e nível de significância de $95 \%$, totalizando-se 69 indivíduos por grupo. Como não foi possível alcançar a quantidade suficiente de indivíduos no grupo CTLP para o período estudado, a amostra foi considerada de conveniência.

Os CCP foram definidos como cateteres venosos centrais de hemodiálise não tunelizáveis, independentemente do tempo de duração (modelos utilizados: Arrow-Howes ${ }^{\circledR}$ LargeBore $12 \mathrm{Fr} 16$ e $20 \mathrm{~cm}$, Teleflex, Morrisville, Carolina do Norte, EUA; e Duo-Flow ${ }^{\circledR}$ Side x Side 12 Fr 15 e 20 cm, Medcomp, Harleysville, Pensilvânia, EUA). Os CTLP foram definidos como cateteres tunelizáveis de duplo lúmen (modelo Hemo-Cath ${ }^{\circledR}$ LT 12,5 Fr 28 e 32 cm, Medcomp). Os CCP foram inseridos por nefrologista ou por cirurgião vascular em sala de procedimento sem auxílio de fluoroscopia. Somente os CCP implantados pelo cirurgião tiveram orientação ultrassonográfica, enquanto os nefrologistas utilizaram técnica de marcação anatômica convencional. Todos os CTLP foram implantados por cirurgião vascular em sala de hemodinâmica, por meio de técnica convencional e asséptica sob orientação ultrassonográfica. Não foi administrado nenhum antimicrobiano no peroperatório para prevenção de infecção do acesso, conforme protocolo institucional. Entretanto, todos os pacientes faziam uso ao menos de antiagregação plaquetária (ácido acetilsalicílico $100 \mathrm{mg} /$ dia ou bissulfato de clopidogrel $75 \mathrm{mg} /$ dia) ou anticoagulação oral. Não foi possível rastrear possíveis falhas de aderência no uso dos antiagregantes ou anticoagulantes ao longo do acompanhamento. Os dados foram extraídos a partir de banco de dados dedicado ao serviço de hemodiálise (NefroSys ${ }^{\circledR}$, Porto Alegre, RS) e inseridos em planilha eletrônica. A alimentação do banco de dados eletrônico foi feita de forma contínua por equipe multiprofissional. Foram incluídos todos os pacientes submetidos ao implante de cateter de curta ou longa 
permanência que apresentaram prontuários completos e excluídos aqueles com cateteres implantados em veia femoral. Somente o primeiro implante de cada tipo de cateter foi considerado para estudo. Pacientes cujos CCP foram substituídos por CTLP ao longo do estudo foram incluídos na análise de duração do cateter duas vezes. Para a análise de sobrevida global, somente os casos em que os CCP se tornaram o único tipo de acesso utilizado foram incluídos. As falhas técnicas relacionadas aos implantes dos cateteres que não permitiram o seu uso não foram contabilizadas como acesso realizado e, consequentemente, eles não foram elegíveis a ser incluídos no estudo.

Foram coletadas informações epidemiológicas (idade, sexo, raça, escolaridade, renda familiar, tipo de provedor de saúde), data de implante e retirada do cateter, motivo de retirada, complicações, fatores de risco presentes [hipertensão arterial sistêmica (HAS), diabetes melito, dislipidemia, tabagismo], presença de infeção e patógeno identificado e data de óbito. HAS foi considerada como pressão arterial maior que $140 \times 90 \mathrm{~mm} \mathrm{Hg}$ ou uso de hipotensivos, diabetes melito como glicemia de jejum $>126 \mathrm{mg} / \mathrm{dL}$ ou glicemia pós-prandial $>200 \mathrm{mg} / \mathrm{dL}$ ou uso contínuo de hipoglicemiantes, dislipidemia como LDL $>130 \mathrm{mg} / \mathrm{dL}$ ou uso de estatina, e tabagismo como qualquer uso contínuo de pelo menos um cigarro por dia. Os dados de cultura aeróbia foram utilizados por meio da ponta do cateter, seccionada no momento da retirada em via estéril, e o resultado foi dado como positivo se $>100 \mathrm{UFC} / \mathrm{mm}^{2}$ fossem isoladas.

A estatística descritiva foi dada pela média ou mediana e porcentagem. A estatística inferencial foi dada pelo teste $t$ para amostras independentes bicaudal, teste de Qui-quadrado $\left(X^{2}\right)$ ou teste de
Fisher, dependendo das variáveis analisadas, após a identificação de outliers pelo método ROUT. A comparação da taxa de duração do cateter foi realizada pela curva de sobrevida pelo método de Kaplan-Meier, com cálculo do log-rank. Foi calculada a taxa global de infecção por 1.000 dias de cateter e a taxa de mortalidade por 1.000 pacientes/ano. Foi utilizado o software estatístico GraphPad Prism v.8, com significância estatística se $\mathrm{p}<0,05$ e índice de confiança de $95 \%$.

\section{RESULTADOS}

Cento e quinze pacientes foram analisados, sendo que 67 foram submetidos ao implante de CCP e 48, de CTLP (Figura 1). Sessenta por cento eram do sexo masculino e a média de idade foi de 62 anos. A veia jugular interna direita foi o principal sítio de punção, tendo sido usada em $85,1 \%$ dos CCP e em $79,2 \%$ dos CTLP. Houve uma prevalência muito grande de HAS $(95,7 \%)$ e diabetes melito (47\%), enquanto dislipidemia $(23,5 \%)$ e tabagismo $(12,2 \%)$ foram menos frequentes. Não houve diferença quanto ao índice de massa corporal (IMC) em nenhum dos grupos. Dos 67 casos de CCP, 20 (29,9\%) foram inseridos por cirurgião vascular. A Tabela 1 mostra as características clínicas e frequência dos sítios de acesso utilizados em ambos os grupos. Todos os pacientes foram acompanhados neste estudo até o momento da retirada do cateter ou do óbito.

O tempo de permanência dos cateteres foi significativamente diferente, sendo que os CCP tiveram uma mediana de 50 dias e os CTLP, de 112 dias [IC (intervalo de confiança) 95\%, CCP: 45,1-63,3 dias versus CTLP: 101,7-159,7 dias; logrank, $\mathrm{p}<0,0001$ ] (Figura 2). Houve 21 óbitos entre

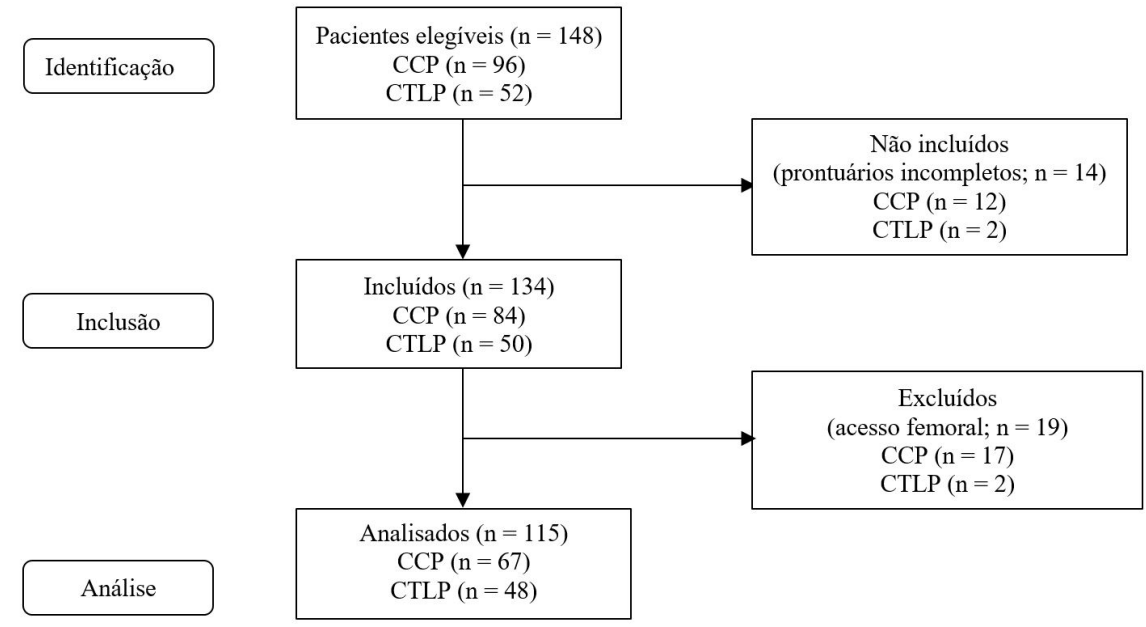

Figura 1. Diagrama de fluxo dos pacientes inseridos no estudo. CCP = cateteres não tunelizáveis de curta permanência; CTLP = cateteres tunelizáveis de longa permanência. 
Tabela 1. Características epidemiológicas e fatores de risco presentes na amostra e em cada um dos subgrupos analisados.

\begin{tabular}{|c|c|c|c|c|}
\hline Fatores de risco & $\begin{array}{l}\text { Total } \\
\mathrm{n}(\%)\end{array}$ & $\begin{array}{c}\text { CCP } \\
\mathrm{n}(\%)\end{array}$ & $\begin{array}{l}\text { CTLP } \\
\mathrm{n}(\%) \\
\end{array}$ & $\mathrm{p}$ \\
\hline Idade (anos) & $62,2( \pm 15,0)^{*}$ & $58,2( \pm 14,5)^{*}$ & $67,8( \pm 13,9)^{*}$ & $0,0006^{5}$ \\
\hline \multicolumn{5}{|l|}{ Sexo } \\
\hline Masculino & $69(60)$ & $44(63,8)$ & $25(36,2)$ & $0,14^{\epsilon}$ \\
\hline Feminino & $46(40)$ & $23(50)$ & $23(50)$ & \\
\hline IMC & $24( \pm 5,6)^{*}$ & $24,2( \pm 5,8)^{*}$ & $23,7( \pm 5,4)^{*}$ & $0,69^{5}$ \\
\hline \multicolumn{5}{|l|}{ Comorbidades } \\
\hline Hipertensão & $110(95,7)$ & $63(94)$ & $47(98)$ & $0,39^{+}$ \\
\hline Diabetes melito & $54(47)$ & $30(45)$ & $24(50)$ & $0,58^{\epsilon}$ \\
\hline Dislipidemia & $27(23,5)$ & $9(13,4)$ & $18(37,5)$ & $0,003^{\dagger}$ \\
\hline Tabagismo & $14(12,2)$ & $11(16,4)$ & $3(6,3)$ & $0,14^{+}$ \\
\hline Tipo de acesso & & & & $0,18^{\epsilon}$ \\
\hline Femoral & $4(3,5)$ & $3(4,5)$ & $1(2,1)$ & \\
\hline Jugular D & $95(82,6)$ & $57(85,1)$ & $38(79,2)$ & \\
\hline Jugular E & $14(12,2)$ & $5(7,5)$ & $9(18,8)$ & \\
\hline VAx & $2(1,7)$ & $2(3,0)$ & $0(0)$ & \\
\hline
\end{tabular}

$\mathrm{CCP}$ = cateter não tunelizável de curta permanência; $C T L P$ = cateter tunelizável de longa permanência; $I M C$ = índice de massa corporal; $V A x=$ veia axilar; *desviopadrão; ' teste $t$ de Student; ${ }^{E}$ Qui-quadrado; teste exato de Fisher.

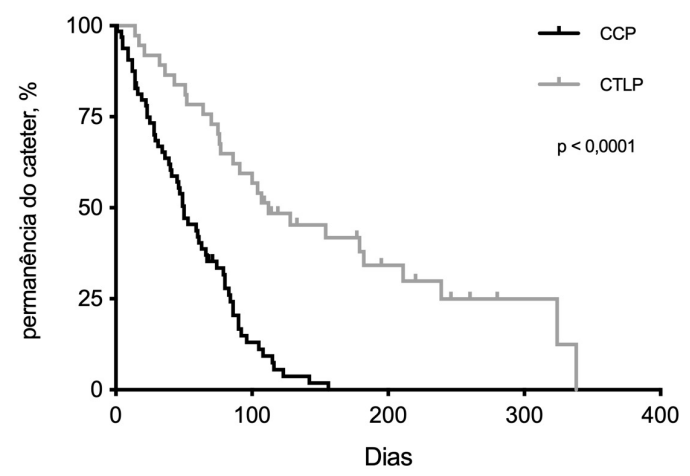

Figura 2. Curva de sobrevida de Kaplan-Meier do tempo de permanência entre os cateteres não tunelizáveis de curta permanência (CCP) e os cateteres tunelizáveis de longa permanência (CTLP), implantados ao longo de um período máximo de 338 dias de observação. Foi observada diferença significativa entre os grupos (teste log-rank).

os usuários de CCP e 20 entre os de CTLP durante todo o período de acompanhamento. A mortalidade operatória (30 dias) foi de 1,5\% para o grupo CCP, enquanto não foi observado óbito no grupo CTLP no período mencionado. Em relação à mortalidade global, a análise de sobrevida não identificou diferença entre os grupos (mediana de sobrevida CCP: 2,87 anos versus CTLP: 3,34 anos; log-rank, $\mathrm{p}=0,68$ ), sendo observada uma mortalidade bruta no primeiro ano de $20 \%$ para os CCP e $23,5 \%$ para os CTLP (Figura 3 ).

As causas de retirada do cateter foram classificadas como obtenção de acesso definitivo (confecção de fístula arteriovenosa ou implante de CTLP), infecção (intraluminal ou sistêmica) e causa mecânica (trombose,

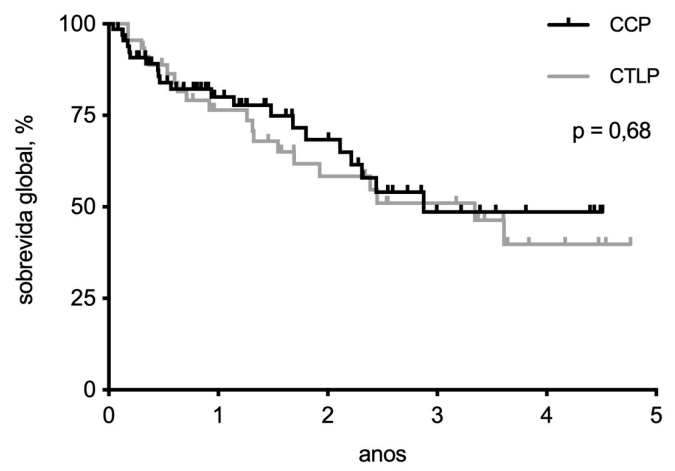

Figura 3. Curva de Kaplan-Meier para a sobrevida global dos pacientes submetidos ao implante de cateteres de curta permanência (CCP) e de cateteres tunelizáveis de longa permanência (CTLP), ao longo de 4,8 anos de observação. Não foi observada diferença significativa entre os grupos (teste log-rank).

acotovelamento ou exteriorização). No total, $63 \mathrm{CCP}$ foram retirados $(94,0 \%)$, enquanto $40(83,4 \%)$ dos CTLP foram removidos no período de observação (Tabela 2). Alguns pacientes foram a óbito com o cateter implantado; portanto, não foram incluídos como perda do acesso. A análise de sobrevida intragrupo revelou uma maior tendência de retirada dos CCP nos casos de infecção ou causa mecânica em detrimento da obtenção de acesso definitivo (log-rank; $\mathrm{p}=0,051)$. No caso dos CTLP, a principal causa de retirada foi complicação mecânica em relação às demais (log-rank; $\mathrm{p}=0,002$ ) (Figura 4). Não foi realizada nenhuma tentativa de fibrinólise do $\mathrm{CVCH}$ por meio de injeção 
Tabela 2. Número da casos de retirada de cateteres não tunelizáveis de curta permanência (CCP) ou cateteres tunelizáveis de longa permanência (CTLP) conforme a causa. Não foi observada diferença estatística entre os grupos $\left(X^{2}: 3,108 ; 2 g l ; p=0,21\right)$.

\begin{tabular}{|c|c|c|c|c|}
\hline \multirow{2}{*}{ Causas de retirada de cateter } & \multicolumn{2}{|c|}{ CCP } & \multicolumn{2}{|c|}{ CTLP } \\
\hline & $\mathrm{n}$ & $\%$ & $\mathrm{n}$ & $\%$ \\
\hline Infecção & 11 & 16,4 & 12 & 25,0 \\
\hline Causas mecânicas & 22 & 32,8 & 15 & 31,3 \\
\hline Acesso definitivo & 30 & 44,8 & 13 & 27,1 \\
\hline Total & 63 & 94,0 & 40 & 83,4 \\
\hline
\end{tabular}
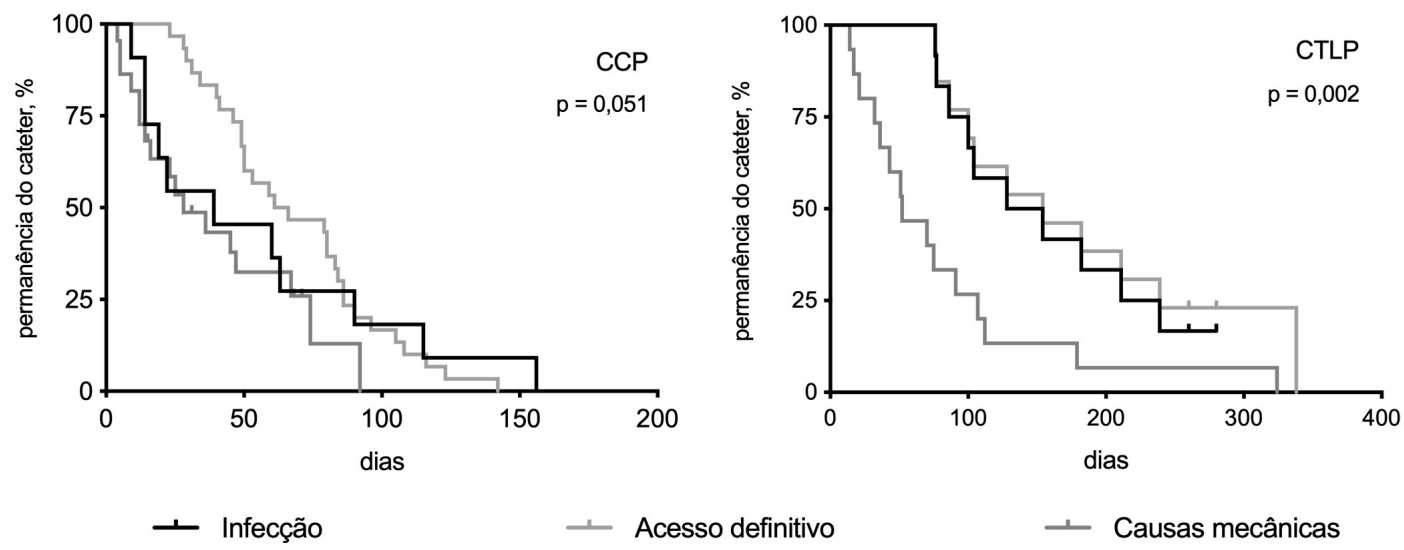

- Acesso definitivo

ᄂ Causas mecânicas

Figura 4. Curva de Kaplan-Meier do tempo de permanência dos cateteres em relação ao motivo de retirada [infecção do cateter; realização de acesso definitivo, seja fístula arteriovenosa ou implante de cateter tunelizável de longa permanência (CTLP); ou causas mecânicas como trombose, acotovelamento ou exteriorização]. No grupo CCP (cateter não tunelizável de curta permanência), não foi observada diferença significativa, porém o implante de acesso definitivo foi a causa menos comum de retirada. No grupo CTLP, as causas mecânicas foram o principal motivo de retirada ou troca do cateter.

de r-TPA ou heparina, ou de remoção mecânica de fibrina em ponta de cateter.

A infecção relacionada ao cateter ocorreu em 57 casos de CCP $(85,1 \%)$ e em 34 (70,8\%) CTLP $\left(X^{2}=3,4 ; p=0,063\right)$. Somente dez casos de infecção em CCP foram submetidos à cultura de cateter, sendo identificado o patógeno em somente quatro casos (três Staphylococcus sp e um Acinetobacter multirresistente), enquanto nos CTLP, 14 foram submetidos à cultura, com identificação de 6 casos (quatro Staphylococcus sp, um Enterobacter cloacae e uma Serratia narcences). Não houve dados suficientes para estudo sobre infecção de pele, de ponta de cateter, sepse ou endocardite. Em todos os casos de infecção de cateter, submetidos ou não à retirada, foi administrada antibioticoterapia sistêmica ou intraluminal, a critério do médico assistente.

Ao analisar individualmente os diferentes fatores potencialmente relacionados à ocorrência de infecção (sexo, escolaridade, convênio médico, renda familiar e raça), somente a baixa renda apresentou resultado estatisticamente significante $\left(X^{2}=35,4 ; \mathrm{p}<0,0001\right)$ (Tabela 3). A taxa de infecção por 1.000 dias foi maior nos CCP em relação aos CTLP (16,7 eventos/1.000 dias versus 7,0 eventos/1.000 dias). A mortalidade acumulada foi de 210 óbitos por 1.000 pacientes/ano para os CCP e 239 óbitos/1.000 pacientes/ano para os CTLP.

\section{DISCUSSÃO}

Este trabalho observou um tempo de duração dos CTLP correspondente a pouco mais que dobro dos CCP (mediana de sobrevida de 50 versus 112 dias) e baixa renda como fator associado à perda do acesso devido à infeção relacionada ao cateter, em serviço de hemodiálise de hospital de ensino.

Estima-se que, no Brasil, no ano de 2016, havia mais de 122 mil pacientes em terapia de substituição renal, o equivalente a uma taxa de 596 pacientes por milhão da população, estando a maioria (92\%) em hemodiálise, o que indica um aumento gradual ao longo dos anos ${ }^{2}$. Estimou-se que 20,5\% desses pacientes faziam uso de cateter de hemodiálise (aprox. 9,4\% CCP e 11,2\% CTLP). O presente trabalho não estudou esse tipo de prevalência por ser longitudinal; entretanto, dados internos do serviço apontam para prevalências de cateteres em torno de 20 a $30 \%$ ao longo dos últimos 3 anos (não publicados). 
Tabela 3. Fatores de risco potencialmente relacionados à ocorrência de infecção de cateter, independentemente do tipo.

\begin{tabular}{|c|c|c|c|c|}
\hline Fatores de Risco & $\begin{array}{c}\text { Infecção } \\
\mathrm{n}(\%)\end{array}$ & $\begin{array}{c}\text { Sem Infecção } \\
\mathrm{n}(\%)\end{array}$ & $\mathrm{p}$ & $\begin{array}{c}\text { Valor do } \\
\text { teste }\end{array}$ \\
\hline \multicolumn{5}{|l|}{ Sexo } \\
\hline Masculino & $13(54)$ & $56(62)$ & $0,64^{+}$ & na \\
\hline Feminino & $11(46)$ & $35(38)$ & & \\
\hline \multicolumn{5}{|l|}{ Renda } \\
\hline Sem renda & $6(25)$ & $5(5)$ & & \\
\hline Até $1 \mathrm{SM}$ & $17(71)$ & $21(23)$ & & \\
\hline$(1-5] \mathrm{SM}$ & $1(4)$ & $58(64)$ & $<0,0001^{\varepsilon}$ & 35,38 \\
\hline$(5-10]$ SM & $0(0)$ & $6(7)$ & & \\
\hline (10-20] SM & $0(0)$ & $1(1)$ & & \\
\hline \multicolumn{5}{|l|}{ Escolaridade } \\
\hline Analfabeto & $7(29)$ & $9(10)$ & $0,10^{E}$ & 9,075 \\
\hline $1^{\circ}$ grau incompleto & $10(42)$ & $57(63)$ & & \\
\hline $1^{\circ}$ grau completo & $2(8)$ & $4(4)$ & & \\
\hline $2^{\circ}$ grau incompleto & $0(0)$ & $3(3)$ & & \\
\hline $2^{\circ}$ grau completo & $2(8)$ & $12(13)$ & & \\
\hline Superior & $3(13)$ & $6(7)$ & & \\
\hline \multicolumn{5}{|l|}{ Raça/Etnia } \\
\hline Branco & $18(75)$ & $69(76)$ & & \\
\hline Pardo & $2(8)$ & $7(8)$ & $0,99^{£}$ & 0,01 \\
\hline Negro & $4(17)$ & $15(16)$ & & \\
\hline \multicolumn{5}{|l|}{ Provedor } \\
\hline Sistema público & $22(92)$ & $86(95)$ & $0,63^{+}$ & na \\
\hline Convênio/privado & $2(8)$ & $5(5)$ & & \\
\hline
\end{tabular}

$\mathrm{SM}$ = salário mínimo; na = não aplicável; ${ }^{\mathrm{E}} \mathrm{Qui}$-quadrado; ${ }^{\dagger}$ teste exato de Fisher.

As características demográficas gerais dos pacientes dialíticos apresentaram resultados semelhantes à literatura, com maior prevalência do sexo masculino $(60 \% \text { do total })^{9}$. A HAS, entretanto, estava presente na quase totalidade dos pacientes como doença de base, o que contrasta com outros estudos nacionais, em que essa taxa gira em torno de 40 a $60 \% \%^{5,9}$. Essa diferença ocorreu uma vez que a HAS nesses trabalhos foi descrita como etiologia para a doença renal terminal, e não como comorbidade presente ao longo do estudo. Interessante notar que os pacientes submetidos ao implante de CTLP se encontravam em faixa etária aproximadamente 10 anos maior que os de CCP, o que pode indicar a dificuldade na confecção de um acesso autólogo definitivo nesses casos. Essa discrepância de idade já foi observada isoladamente em outros estudos ${ }^{10,11}$.

O sítio ideal de implante do cateter venoso central ainda é tema de discussão. Embora estudos anteriores tenham mostrado uma menor taxa de infeção relacionada ao acesso jugular em relação ao femoral, uma metanálise publicada em 2012 com mais de 17 mil cateteres centrais simples implantados em ambiente hospitalar não evidenciou diferença [risco relativo (RR) 1,35; IC 95\% 0,84-2,19] ${ }^{12}$. Apesar disso, entende-se que, em relação aos cateteres de hemodiálise, devido ao subgrupo específico de usuários, a preferência pelo acesso jugular está associada a menor infeção e complicações de punção, além de menor incidência de estenose de veia central ${ }^{13,14}$. A lateralidade também é importante, uma vez que cateteres implantados à direita possuem menos disfunção ou infecção do que à esquerda ${ }^{15}$. No presente trabalho, a prevalência de uso de veia jugular interna direita como sítio de acesso ocorreu em mais de $80 \%$ dos casos.

As taxas de sobrevida de cateter observadas neste estudo estão abaixo da maioria dos trabalhos encontrados na literatura, apesar de não haver estudo que compare especificamente os CCP e CTLP. Mandolfo et al. ${ }^{16}$ encontraram uma sobrevida cumulativa de CTLP de $91 \%$ em 1 ano e de $85 \%$ em 4 anos, enquanto Shi et al. ${ }^{17}$ observaram sobrevida de $82 \%$ em 1 ano e de $42 \%$ em 4 anos. Por outro lado, Shingarev et al. ${ }^{14}$ descreveram taxas de perviedade de cateteres bem 
menores, de $54 \%$ em 6 meses e $35 \%$ em 1 ano para veia jugular interna direita. No presente trabalho, não houve CCP com duração maior que 156 dias ou CTLP maior que 338 dias. Essa disparidade deve refletir diferentes realidades na abordagem de preservação dos acessos, desde condições socioeconômicas até intervenções para prevenção e tratamento de complicações infecciosas e mecânicas ${ }^{18}$.

Por outro lado, não foi observada diferença na sobrevida global dos pacientes ao longo dos anos, mesmo que os pacientes do grupo dos CTLP fossem, em média, 10 anos mais velhos. Um estudo publicado em 2018, após observação de 140 mil pacientes usuários de cateter de hemodiálise (como ponte ou não para fístula arteriovenosa), observou sobrevida semelhante ao presente trabalho, porém ressaltou uma maior mortalidade e complicações nesse grupo de pacientes ${ }^{19}$. Um estudo chinês publicado em 2017 também observou sobrevida semelhante ${ }^{17}$. Segundo um estudo brasileiro, a taxa de mortalidade bruta anual é de $18,2 \%$, o que demonstra uma proximidade com a mortalidade do presente estudo ${ }^{2}$.

No presente trabalho, não houve diferenças significativas entre as diferentes causas estudadas de perda de cateter para ambos os tipos. Deve-se ressaltar a alta incidência de complicações mecânicas observadas (tromboses, acotovelamentos, migrações etc.), chegando a um terço dos casos em ambos os grupos. Na análise de sobrevida, entretanto, observamos que a principal causa de perda do CTLP foi justamente a disfunção do cateter. A incidência de disfunção de cateter foi publicada em estudo chinês multicêntrico com 865 pacientes, sendo observados vários fatores independentemente relacionados: residência rural, ausência de anticoagulantes, ausência de exame de imagem de controle, cateter implantado à esquerda, cateter femoral e anemia. No mesmo estudo, observouse uma alta incidência de disfunção: $66 \%$ para $\mathrm{CCP}$ e $45 \%$ para CTLP $(p<0,01)^{20}$. Uma coorte prospectiva envolvendo 154 pacientes durante 16 meses de estudo observou uma incidência 13 vezes maior de disfunção de CCP em comparação com os cateteres tunelizáveis (IC 95\% 2,9-63,0 $)^{21}$.

A complicação dos $\mathrm{CVCH}$ mais relatada e mais temida é a infecção, a qual gera um risco de hospitalização e morte de 2 a 3 vezes maior que os portadores de fistula arteriovenosa ou fístulas protéticas ${ }^{4}$. É associada a diversos fatores de risco que predispõem à elevada taxa de morbimortalidade dos pacientes, com uma incidência estimada de 60 casos por 10.000 admissões. As infecções relacionadas ao acesso vascular podem ser tanto locais (infecções de óstio ou de túnel) como sistêmicas (bacteremias e sepses). Uma vez apresentando sinais clínicos e/ou locais de infecção, deve-se realizar a hemocultura e a administração de antibiótico parenteral ou intraluminal e ser avaliada a remoção cateter. Caso seja removido o cateter, um novo poderá ser inserido, preferencialmente no lado contralateral, após $48 \mathrm{~h}$ de terapia ${ }^{10}$.

Uma coorte multicêntrica canadense, entretanto, observou um risco 9 vezes maior de infecção de CCP e CTLP em relação às FAV, sem notar diferença nas infecções entre os dois tipos de cateteres ${ }^{22}$. É importante que sejam identificadas não somente as infecções sob $\mathrm{o}$ aspecto clínico, mas também microbiológico. $\mathrm{O}$ presente trabalho revelou alta incidência de infecção relacionada ao cateter $(85,1 \%$ nos CCP e $70,8 \%$ nos CTLP), porém a taxa de cultura de ponta do cateter foi desprezível (24 de 91 casos de infecção, ou 26\%). Apesar disso, a taxa de infeção por paciente por ano relatada foi semelhante à do estudo publicado por Sahli et al. (16,6 eventos/1.000 dias) $)^{23}$, e superior à de Murea et al. $(1,97 / 1.000 \text { dias de CTLP })^{24}$, de Wang et al. (12,7 eventos/1.000 dias de cateteres para os CCP e 5,39 eventos/1.000 dias de cateteres para os CTLP $)^{20}$ e de Menegueti et al. ${ }^{7}$ (6,1 infecções de corrente sanguínea/1.000 dias de diálise). Um estudo prospectivo unicêntrico sueco, aplicando protocolo de prevenção de infecção, obteve incidências de colonização, infecção relacionada ao cateter e infecção de corrente sanguínea relacionado ao cateter de $7,0,2,2$ e 0,6 eventos por 1.000 dias de cateteres ${ }^{18}$. Apesar da baixa prevalência de culturas de ponta de cateter, foi possível observar a predominância do $S$. aureus, porém com ressalva para ocorrência de gram-negativos multirresistentes, em concordância com outros estudos nacionais ${ }^{25,26}$.

Entre os fatores estudados potencialmente relacionados à ocorrência de infecção, somente a baixa renda foi estatisticamente relacionada. Noventa e seis por cento dos que apresentaram infecção possuíam até um salário mínimo de renda, enquanto $87 \%$ dos que não apresentaram infecção recebiam de um até cinco salários mínimos. Semelhante a outros estudos, não foi possível relacionar a ocorrência de infecção com baixa escolaridade, sexo, raça ou provedor do tratamento ${ }^{7,22}$. Parece ser importante o impacto que as condições socioeconômicas individuais devem ter na preservação da higiene local, dificuldade de transporte, aderência às medicações, compra de materiais, entre outras ${ }^{27}$.

Entre os pontos negativos do trabalho, podemos enumerar a característica unicêntrica e retrospectiva, a ausência de informações referentes às técnicas de assepsia para implantação dos cateteres, o uso de medicações que alterem o índice de infecção, além da baixa incidência de cultura de ponta de cateter realizada nas suspeitas de infecção, em parte porque 
a coleta de dados dependeu do correto preenchimento dos prontuários hospitalares. Devido a isso, não foi possível a adequada caracterização da flora prevalente nos casos de infecção relacionada ao cateter. Ainda, a amostra por conveniência e com número reduzido no grupo CTLP não permitiu poder de inferência na comparação de durabilidade do acesso venoso central entre os grupos estudados e não foi realizada análise de regressão temporal para definir melhor quais fatores poderiam estar associados à perda do cateter. Por fim, deve-se reconhecer a heterogeneidade da amostra, na qual profissionais distintos realizaram o implante de CCP sob diferentes métodos (com e sem orientação ultrassonográfica). O uso do equipamento de ultrassonografia por parte do nefrologista, sendo este o especialista que mais executa o implante do CCP nos centros de hemodiálise, não é uma realidade no Brasil. Tal heterogeneidade pode ser um ponto positivo do estudo, entretanto, pois confere maior validação externa dos resultados.

Adiminuição das taxas de cateteres e suas complicações infecciosas e mecânicas podem ser alcançadas com a instalação de programas locais de qualidade, com equipe multidisciplinar e metas a serem alcançadas. Tais programas, por exemplo, possibilitaram uma redução na taxa de cateter de 45 para $8 \%$ em centros americanos de diálise que utilizam o Medicare ${ }^{28}$. É evidente que a possibilidade de alcançar tais metas depende também da prevalência de comorbidades (diabetes, por exemplo), idade e quantidade de acessos prévios criados, o que dificulta a confecção de acessos autólogos de alta durabilidade ${ }^{29}$.

\section{CONCLUSÃO}

O tempo de sobrevida dos cateteres de hemodiálise tunelizáveis de longa permanência foi significativamente maior que os de curta permanência, porém ainda assim abaixo dos valores relatados na literatura. Apesar disso, não foi observada diferença de sobrevida dos pacientes utilizando qualquer um dos tipos de cateteres. A análise microbiológica das infecções relacionadas a cateter ainda é pouco realizada, o que impacta na sua real incidência. A baixa renda esteve associada a maior taxa de infecção, o que reflete a realidade de um serviço de hemodiálise de país em desenvolvimento.

\section{REFERÊNCIAS}

1. Ravani P, Palmer SC, Oliver MJ, et al. Associations between hemodialysis access type and clinical outcomes: a systematic review. J Am Soc Nephrol. 2013;24(3):465-73. http://dx.doi.org/10.1681/ ASN.2012070643. PMid:23431075.
2. Sesso RC, Lopes AA, Thomé FS, Lugon JR, Martins CT. Brazilian chronic dialysis survey 2016. J Bras Nefrol. 2017;39(3):261-6. http:// dx.doi.org/10.5935/0101-2800.20170049. PMid:29044335.

3. Grothe C, Belasco AGS, Bittencourt ARC, Vianna LAC, Sesso RCC, Barbosa DA. Incidência de infecção da corrente sanguínea nos pacientes submetidos à hemodiálise por cateter venoso central. Rev Lat Am Enfermagem. 2010;18(1):73-80. http://dx.doi.org/10.1590/ S0104-11692010000100012. PMid:20428700.

4. Böhlke M, Uliano G, Barcellos FC. Hemodialysis catheter-related infection: prophylaxis, diagnosis and treatment. J Vasc Access. 2015;16(5):347-55. http://dx.doi.org/10.5301/jva.5000368. PMid:25907773.

5. Bonfante GM, Gomes IC, Andrade EIG, Lima EM, Acurcio FA, Cherchiglia ML. Duration of temporary catheter use for hemodialysis: an observational, prospective evaluation of renal units in Brazil. BMC Nephrol. 2011;12(1):63. http://dx.doi.org/10.1186/14712369-12-63. PMid:22093280.

6. Miller LM, Clark E, Dipchand C, et al. Hemodialysis tunneled catheter-related infections. Can J Kidney Health Dis. 2016;3. PMid:28270921.

7. Menegueti MG, Betoni NC, Bellissimo-Rodrigues F, Romão EA. Central venous catheter-related infections in patients receiving short-term hemodialysis therapy: incidence, associated factors, and microbiological aspects. Rev Soc Bras Med Trop. 2017;50(6):783-7. http://dx.doi.org/10.1590/0037-8682-0438-2017. PMid:29340455.

8. Schwanke AA, Danski MTR, Pontes L, Kusma SZ, Lind J. Central venous catheter for hemodialysis: incidence of infection and risk factors. Rev Bras Enferm. 2018;71(3):1115-21. http://dx.doi. org/10.1590/0034-7167-2017-0047. PMid:29924154.

9. Ferreira $V$, Andrade D. Cateter para hemodiálise: retrato de uma realidade. Medicina (B Aires). 2007;40(4):582. http://dx.doi. org/10.11606/issn.2176-7262.v40i4p582-588.

10. Martín-Peña A, Márquez RL, Guerrero MJM, et al. Tunneled hemodialysis catheter-related bloodstream infections: a prospective multicenter cohort study from Spain. J Vasc Access. 2012;13(2):23945. http://dx.doi.org/10.5301/jva.5000034. PMid:22266591.

11. Fram D, Okuno MFP, Taminato $M$, et al. Risk factors for bloodstream infection in patients at a Brazilian hemodialysis center: a case-control study. BMC Infect Dis. 2015;15(1):158. http://dx.doi.org/10.1186/ s12879-015-0907-y. PMid:25879516.

12. Marik PE, Flemmer $M$, Harrison W. The risk of catheter-related bloodstream infection with femoral venous catheters as compared to subclavian and internal jugular venous catheters: a systematic review of the literature and meta-analysis. Crit Care Med. 2012;40(8):2479-85. http://dx.doi.org/10.1097/ CCM.0b013e318255d9bc. PMid:22809915.

13. Clark EG, Barsuk JH. Temporary hemodialysis catheters: recent advances. Kidney Int. 2014;86(5):888-95. http://dx.doi.org/10.1038/ ki.2014.162. PMid:24805107.

14. Shingarev R, Barker-Finkel J, Allon M. Natural history of tunneled dialysis catheters placed for hemodialysis initiation. J Vasc Interv Radiol. 2013;24(9):1289-94. http://dx.doi.org/10.1016/j. jvir.2013.05.034. PMid:23871694.

15. Engstrom BI, Horvath JJ, Stewart JK, et al. Tunneled internal jugular hemodialysis catheters: impact of laterality and tip position on catheter dysfunction and infection rates. J Vasc Interv Radiol. 2013;24(9):1295-302. http://dx.doi.org/10.1016/j.jvir.2013.05.035. PMid:23891045.

16. Mandolfo S, Acconcia P, Bucci R, et al. Hemodialysis tunneled central venous catheters: five-year outcome analysis. J Vasc Access. 2014;15(6):461-5. http://dx.doi.org/10.5301/jva.5000236. PMid:24811590. 
17. Shi M, Cui T, Ma L, Zhou L, Fu P. Catheter failure and mortality in hemodialysis patients with tunneled cuffed venous catheters in a single center. Blood Purif. 2017;43(4):321-6. http://dx.doi. org/10.1159/000455062. PMid:28135701.

18. Hammarskjöld F, Berg S, Hanberger H, Taxbro K, Malmvall B-E. Sustained low incidence of central venous catheter-related infections over six years in a Swedish hospital with an active central venous catheter team. Am J Infect Control. 2014;42(2):122-8. http://dx.doi. org/10.1016/j.ajic.2013.09.023. PMid:24485369.

19. Arhuidese IJ, Orandi BJ, Nejim B, Malas M. Utilization, patency, and complications associated with vascular access for hemodialysis in the United States. J Vasc Surg. 2018;68(4):1166-74. http://dx.doi. org/10.1016/j.jvs.2018.01.049. PMid:30244924.

20. Wang K, Wang P, Liang X, Lu X, Liu Z. Epidemiology of haemodialysis catheter complications: a survey of 865 dialysis patients from 14 haemodialysiscentres in Henan province in China. BMJ Open. 2015;5(11):e007136. http://dx.doi.org/10.1136/bmjopen-2014-007136. PMid:26589425.

21. Mendu ML, May MF, Kaze AD, et al. Non-tunneled versus tunneled dialysis catheters for acute kidney injury requiring renal replacement therapy: a prospective cohort study. BMC Nephrol. 2017;18(1):351. http://dx.doi.org/10.1186/s12882-017-0760-x. PMid:29202728.

22. Taylor G, Gravel D, Johnston L, Embil J, Holton D, Paton S. Incidence of bloodstream infection in multicenter inception cohorts of hemodialysis patients. Am J Infect Control. 2004;32(3):155-60. http://dx.doi.org/10.1016/j.ajic.2003.05.007. PMid:15153927.

23. Sahli F, Feidjel R, Laalaoui R. Hemodialysis catheter-related infection: rates, risk factors and pathogens. J Infect Public Health. 2017;10(4):403-8. http://dx.doi.org/10.1016/j.jiph.2016.06.008. PMid:27423929.

24. Murea M, James KM, Russell GB, et al. Risk of catheter-related bloodstream infection in elderly patients on hemodialysis. Clin J Am Soc Nephrol. 2014;9(4):764-70. http://dx.doi.org/10.2215/ CJN.07710713. PMid:24651074.

25. Esmanhoto CGU, Taminato MU, Fram DSU, Belasco AGSU, Barbosa DAU. Microrganismos isolados de pacientes em hemodiálise por cateter venoso central e evolução clínica relacionada. Acta Paul Enferm. 2013;26(5):413-20.

26. Zerati $A E$, Wolosker N, Luccia N, Puech-Leão P. Cateteres venosos totalmente implantáveis: histórico, técnica de implante e complicações. J Vasc Bras. 2017;16(2):128-39. http://dx.doi. org/10.1590/1677-5449.008216. PMid:29930637.

27. Barbosa DA, Gunji CK, Bittencourt ARC, et al. Co-morbidade e mortalidade de pacientes em início de diálise. Acta Paul Enferm. 2006;19(3):304-9. http://dx.doi.org/10.1590/S0103-21002006000300008.
28. Rosenberry PM, Niederhaus SV, Schweitzer EJ, Leeser DB. Decreasing dialysis catheter rates by creating a multidisciplinary dialysis access program. J Vasc Access. 2018;19(6):569-72. http://dx.doi. org/10.1177/1129729818762977. PMid:29575978.

29. Saleh HM, Tawfik MM, Abouellail H. Prospective, randomized study of long-term hemodialysis catheter removal versus guidewire exchange to treat catheter-related bloodstream infection. J Vasc Surg. 2017;66(5):1427-1431.e1. http://dx.doi.org/10.1016/j. jvs.2017.05.119. PMid:28822660.

Correspondência Seleno Glauber de Jesus-Silva Associação de Integração Social de Itajubá - AISI, Hospital de Clínicas de Itajubá - HCl, Departamento de Cirurgia Vascular e Endovascular Rua Miguel Viana, 420 CEP 37500-080 - Itajubá (MG), Brasil Tel.: (35) 3629-7602/(35) 99931-0929 E-mail: seleno.glauber@hcitajuba.org.br

Informações sobre os autores SGJS - Mestre em Ciências, Universidade Federal de São Paulo (UNIFESP/EPM); Especialista em Cirurgia Vascular e em Radiologia Intervencionista e Angiorradiologia.

JSO e KTFR - Bacharéis em Medicina, Faculdade de Medicina de Itajubá (FMIt).

LAM - Professora, Faculdade de Medicina de Itajubá (FMIt); Especialista em Nefrologia; Supervisora do Programa de Residência Médica de Nefrologia, Hospital de Clínicas de Itajubá, Associação de Integração Social de Itajubá (HCI/AISI).

MAMS - Mestre em Ciências, Universidade Federal de São Paulo

(UNIFESP/EPM); Professora, Faculdade de Medicina de Itajubá (FMIt); Especialista em Cirurgia Vascular e Ultrassonografia Vascular. AEK - Especialista em Cirurgia Vascular. RSC - Especialista em Cirurgia Vascular e em Radiologia Intervencionista e Angiorradiologia.

Contribuições dos autores Concepção e desenho do estudo: SGJS, JSO, KTFR Análise e interpretação dos dados: SGJS, JSO, KTFR Coleta de dados: ISO, KTFR, LAM Redação do artigo: SGJS, JSO, KTFR Revisão crítica do texto: SGJS, MAMS, AEK Aprovação final do artigo*: SGJS, JSO, KTFR, LAM, MAMS, AEK, RSC Análise estatística: SGJS Responsabilidade geral pelo estudo: SGJS

*Todos os autores leram e aprovaram a versão final submetida ao J Vasc Bras. 